\title{
Paracetamol is an effective drug to use for pain following oral surgery
}

\section{Is paracetamol (acetaminophen) effective in controlling pain after oral surgery?}

\author{
Weil K, Hooper L, Afzal Z, et al. \\ Paracetamol for pain relief after surgical removal of lower wisdom \\ teeth. Cochrane Database Syst Rev 2007; issue 3
}

\begin{abstract}
Data sources Studies were identified using the Cochrane trials registers of the Oral Health Group and of the Pain, Palliative and Supportive Care Group, along with the Cochrane Central Register of Controlled Trials, Medline, Embase and the Current Controlled Trials Register. Handsearching included several dental journals as well as the bibliographies of relevant clinical trials and review articles for studies outside the journals searched by hand. Authors of the randomised controlled trials (RCT) identified and manufacturers of analgesic pharmaceuticals were contacted in an attempt to identify unpublished or ongoing RCT. No language restriction was applied.
\end{abstract}

Study selection Randomised, parallel-group, placebo-controlled, double-blind clinical trials of paracetamol (acetaminophen) for acute pain following third molar surgery were included.

Data extraction and synthesis All trials identified were scanned independently and in duplicate by two review authors. Any disagreements were resolved by discussion or, if necessary, a third review author was consulted. The proportion of patients who recorded at least $50 \%$ pain relief was calculated for both paracetamol and placebo. The number of patients experiencing adverse events, and/ or the total number of adverse events reported were analysed.

Results Twenty-one trials met the inclusion criteria. A total of 2048 people were initially enrolled in the trials (1148 took paracetamol and 892 the placebo) and of these 1968 (96\%) were included in the metaanalysis (1133 took paracetamol, and 835 the placebo). Paracetamol provided a statistically significant benefit when compared with placebo for pain relief and for pain intensity at both 4 and $6 \mathrm{~h}$. Most studies were found to have moderate risk of bias, with poorly reported allocation concealment being the main problem. The risk ratio for pain relief at $4 \mathrm{~h}$ was 2.85 (95\% confidence interval, 1.89-4.29), and at $6 \mathrm{~h}$ was $3.32(95 \%$ confidence interval, 1.88-5.87). A statistically significant benefit was also found between doses of $<1000 \mathrm{mg}$ and of $1000 \mathrm{mg}$, the higher dose giving greater benefit for pain relief and intensity at both timepoints. There was no statistically significant difference between the number of patients who reported adverse events: this was $19 \%$ in the paracetamol group and $16 \%$ in the placebo group.

Address for correspondence: Luisa Fernandez, Group Co-ordinator, Cochrane Oral Health Group, MANDEC, 3rd Floor Dental Hospital, University of Manchester, Higher Cambridge Street, Manchester M15 6FH, UK.

E-mail: luisa.fernandez@manchester.ac.uk
Conclusions Paracetamol is an effective drug to use for postoperative pain following oral surgery, and the reporting of adverse events shows it to be a safe drug: the number-needed-to-treat (NNT) to benefit is three for $1000 \mathrm{mg}$ of paracetamol at $6 \mathrm{~h}$ and the NNT to harm is 33 . It is most effective at a $1000 \mathrm{mg}$ dose, and can be taken at 6-hourly intervals without compromising safety. It could be considered more readily by dentist and patients both as a first-choice analgesic, or to be taken alternately with other analgesics such as nonsteroidal anti-inflammatory drugs.

\section{Commentary}

There is an explosion in the availability of clinical information - there are over 600000 articles published in the biomedical literature each year. Even diligent readers who read two articles every day would be more than 800 years behind the literature within 1 year. Time is scarce: life is too short to read bad articles. A notable benefit of learning the principles and practice of evidence-based clinical decision-making is the ability to discriminate, ruthlessly when necessary, between articles that should be read carefully, scanned or discarded.

This Cochrane review, despite its excellent quality, should either be scanned or discarded. This is unfortunate. The review process was clearly a major expenditure of human resources. Before expending such effort and resources, it is critical to develop important clinical questions relevant to practice. The clinical question as formulated for this review was not relevant to this reviewer's clinical practice.

Contrary to popular opinion, oral and maxillofacial surgeons do care about postoperative pain control. At face value, the reviewers propose an important question, ie, is paracetamol effective in controlling post-operative pain in people who have had oral surgery? A more careful reading of the review suggests that the following question is being addressed, "Do people who take paracetamol after extraction of mandibular third molars (M3) have improved postoperative pain control compared with patients taking placebo?" Comparing paracetamol with a placebo control is an irrelevant clinical comparison. People who have M3 removed, assuming average difficulty, are rarely, if ever, discharged with the advice to "take no pain medicine." Although this review clearly demonstrates that paracetamol is better than nothing, nothing is an unreasonable treatment alternative for postoperative pain.

In any comparative study or review, careful attention needs to be paid to the choice of control. In most textbook examples, the control is a placebo. Unfortunately, having a placebo chosen as the control may result in a technically excellent study that is practically useless. Practical clinical trials choose a control that is the standard or usual treatment. In the setting of operations associated with moderate to severe postoperative pain, a placebo control is a poor choice as the comparison. This review would be much more valuable if the control choices were other standard/ usual analgesics or regimens such as ibuprofen or narcotic combinations (acetoaminophen and hydrocodone) or preoperative versus postoperative analgesic dosing. It is 
possible that the proposed research question evolved in response to the quality of the available data. If the available data result in an irrelevant research question, my advice would be to abort the exercise and spend the time developing new questions.

This review creates the conundrum of what to do with a technically excellent review that addresses a clinically irrelevant question. There are two interesting findings, however. For clinicians who routinely use paracetamol for postoperative pain control following M3 removal, this review provides good information regarding the dose and frequency of administration. The other interesting finding was the surprisingly high frequency of adverse reactions reported to paracetamol, a medication readily available to the public, and to placebo, $19 \%$ and $16 \%$, respectively. This review, taken jointly with the above comments regarding the development of

\section{Comment from Paul Coulthard on behalf of the authors}

Even though this commentator doubts the clinical relevance of the question posed in this Cochrane review in his practice, we believe that it may be of interest to many other clinicians, and especially to oral and maxillofacial surgeons. We understand that the appropriateness of using a placebo arm in a trial has been much debated over time and we chose at the outset of writing this review to use this comparison because there is much merit in doing so. Contrary an important clinical question, serves as an excellent model for other researchers interested in developing systematic reviews of postoperative pain control.

\section{Practice point}

This review provides useful information on the dose and frequency of paracetamol for pain relief following third molar removal, but it should be noted that $19 \%$ of patients reported adverse events.

\section{Tom Dodson}

Oral and Maxillofacial Surgery Department, Harvard University School of Dental Medicine, Boston, USA

Evidence-Based Dentistry (2007) 8, 5-6. doi:10.1038/sj.ebd.6400510

to the reviewers' suspicion, we did not evolve this position during the writing of the review because of any difficulties in availability of data. Placebos have powerful effects and there are therefore many scientific reasons to want to use a placebo in a trial and few reasons not to use one. The placebo comparison also facilitates random assignment and blinding.

We thank the reviewer for his positive comments and in particular the acknowledgment of the high quality of the review. 